\title{
Variable polarization measured in the prompt emission of GRB 041219A using IBIS on board INTEGRAL
}

\author{
Diego Götz ${ }^{1}$, Philippe Laurent ${ }^{2}$, and François Lebrun ${ }^{2}$ \\ CEA Saclay, DSM/Irfu/Service d'Astrophysique, F-91191, Gif sur Yvette, France \\ diego.gotz@cea.fr \\ Frédéric Daigne ${ }^{3}$, and Željka Bošnjak \\ Institut d'Astrophysique de Paris, UMR 7095 Université Pierre et Marie Curie-Paris 6 - CNRS, 98 bis \\ boulevard Arago, 75014 Paris, France
}

\begin{abstract}
Polarization measurements provide direct insight into the nature of astrophysical processes. Unfortunately, only a few instruments are available for this kind of measurements at $\gamma$-ray energies, and the sources need to be very bright. Gamma-Ray Bursts (GRBs) are ideal candidates due to their large flux over limited time intervals, maximizing the available signal-to-noise ratio. To date a few polarization measurements have been reported, claiming of a high degree of polarization in the prompt emission of GRBs but with low statistical evidence.

We used the IBIS telescope on board the INTEGRAL satellite to measure the polarization of the prompt $\gamma$-ray emission of the long and bright GRB 041219A in the 200-800 keV energy band. We find a variable degree of polarization ranging from less than $4 \%$ over the first peak to $43 \pm 25 \%$ for the whole second peak. Time resolved analysis of both peaks indicates a high degree of polarization, and the null average polarization in the first peak can be explained by the rapid variations observed in the polarization angle and degree.

Our results are consistent with different models for the prompt emission of GRBs at these energies, but they favor synchrotron radiation from a relativistic outflow with a magnetic field which is coherent on an angular size comparable with the angular size of the emitting region $(\sim 1 / \Gamma)$. Indeed this model has the best capabilities to maintain a high polarization level, and to produce the observed variability.
\end{abstract}

Subject headings: gamma rays: bursts - polarization — gamma rays: observations

\section{Introduction}

Gamma Ray Bursts (GRBs) are powerful flashes of $\gamma$-ray radiation appearing at random directions on the sky, and lasting from a fraction to hundreds of seconds. Some of them are firmly associated with supernovae of type $\mathrm{Ib} / \mathrm{c}$, and the

\footnotetext{
${ }^{1}$ Astrophysique Interactions Multi-échelles (AIM), CNRS

${ }^{2}$ Astroparticules et Cosmologie (APC) - 10, rue Alice Domon et Léonie Duquet, F-75205, Paris Cedex 13, France

${ }^{3}$ Institut Universitaire de France
}

general picture is that bursts longer than $1 \mathrm{~s}$ are associated with the death of massive $\left(\gtrsim 30 \mathrm{M}_{\odot}\right)$ stars (for a recent review on GRBs, see Mészáros 2006). The isotropic equivalent energy radiated during the prompt phase is of the order of $10^{51}$ to $10^{54} \mathrm{ergs}$ and is believed to originate from a highly relativistic outflow $(\Gamma \gtrsim 100)$ ejected by the central source. The precise content of this jet, and especially its magnetization, as well as the details of the mechanism leading to the $\gamma$-ray emission are still uncertain. Models range from unmagnetized fireballs where the observed emission could 
be produced by relativistic electrons accelerated in internal shocks propagating within the outflow (Rees \& Mészáros 1994), to pure electromagnetic outflows where the radiated energy comes from magnetic dissipation (Lyutikov 2006). Intermediate cases with mildly magnetized outflows are of course possible (e.g. Spruit et al. 2001). Even in the case of an unmagnetized fireball, a local magnetic field in the emission region, generated by the shocks, is necessary if the dominant process is synchrotron radiation from relativistic electrons. In the case of a mildly magnetized outflow, such additional shock-generated magnetic field could also be present. The present situation is that neither the global magnetization of the outflow, nor the local intensity of the field are well constrained by the observations.

Polarization measurements of the prompt phase of GRBs can shed new light on the strength and scale of magnetic fields, as well as on the radiative mechanisms at work. Locally, the synchrotron emission has a high degree of linear polarization, that however could be reduced by relativistic effects in a jet. In the case where the magnetic field is mainly transverse and highly ordered, i.e. has a coherence scale which is larger than the typical size $\sim R / \Gamma$ of the visible part of the emitting region, the detected signal can still be highly polarized. On the other hand, in the case of a random field or an ordered magnetic field parallel to the expansion velocity, the polarization of the detected signal should vanish, except for the peculiar condition of a jet observed slightly off-axis (Waxman 2003).

To date only a few polarization measurements are available for GRBs. Coburn \& Boggs (2003) reported a high degree of polarization, $\Pi=80 \% \pm 20 \%$, for GRB 021206. However, successive reanalysis of the same data set could not confirm this claim, reporting a degree of polarization compatible with zero (Rutledge \& Fox 2004; Wigger et al. 2004). Willis et al. (2005) reported a strong polarization signal in GRB 930131 $(\Pi>35 \%)$ and GRB $960924(\Pi>50 \%)$, but this result could not be statistically constrained. GRB 041219A is the longest and brightest GRB localized by INTEGRAL (Winkler et al. 2003) to date (Vianello et al. 2009). McGlynn et al. (2007), using the data of the INTEGRAL spectrometer (SPI; Vedrenne et al. 2003), reported a high degree of polarization of the prompt emission $(\Pi=68 \pm 29 \%)$ for the brightest part of this GRB.

Here we report the polarization measurement of the prompt emission of GRB 041219A, performed using the imager on board INTEGRAL, IBIS (Ubertini et al. 2003).

\section{Observations and analysis}

IBIS is composed of two position sensitive detector layers ISGRI (CdTe, 15-1000 keV; Lebrun et al. 2003), and PICsIT (CsI, $200 \mathrm{keV}$ $10 \mathrm{MeV}$; Labanti et al. 2003), and can be used as a Compton polarimeter (Lei et al. 1997), thanks to the polarization dependency of the differential cross section for Compton scattering

$$
\frac{d \sigma}{d \Omega}=\frac{r_{0}^{2}}{2}\left(\frac{E^{\prime}}{E_{0}}\right)^{2}\left(\frac{E^{\prime}}{E_{0}}+\frac{E_{0}}{E^{\prime}}-2 \sin ^{2} \theta \cos ^{2} \phi\right)
$$

where $r_{0}^{2}$ is the classical electron radius, $E_{0}$ the energy of the incident photon, $E^{\prime}$ the energy of the scattered photon, $\theta$ the scattering angle, and $\phi$ the azimuthal angle relative to the polarization direction. Linearly polarized photons scatter preferentially perpendicularly to the incident polarization vector. Hence by examining the scatter angle distribution of the detected photons

$$
N(\phi)=S\left[1+a_{0} \cos 2\left(\phi-\phi_{0}\right)\right],
$$

one can derive the polarization angle, $P A=$ $\phi_{0}-\pi / 2+n \pi$, and the polarization fraction $\Pi=$ $a_{0} / a_{100}$, where $a_{100}$ is the amplitude expected for a $100 \%$ polarized source derived by Montecarlo simulations (see Forot et al. 2008).

IBIS is a coded mask telescope with a $29^{\circ} \times 29^{\circ}$ field of view at zero sensitivity, and a $8.5^{\circ} \times 8.5^{\circ}$ central region where the sensitivity is maximal and uniform. GRB 041219A was detected at $\sim 3.15^{\circ}$ off-axis. Here we consider the events which interacted once in the upper layer, ISGRI, and once in the lower layer, PICsIT, and whose reconstructed energy lies in the $200 \mathrm{keV}-800 \mathrm{keV}$ range. These events are automatically selected on board through a time coincidence algorithm, whose maximal allowed time window was $3.8 \mu$ s during our observation. A light curve of the Compton events is reported in Fig. 1.

As can be seen, the GRB is clearly detected, but due to its brightness, the available IBIS telemetry 


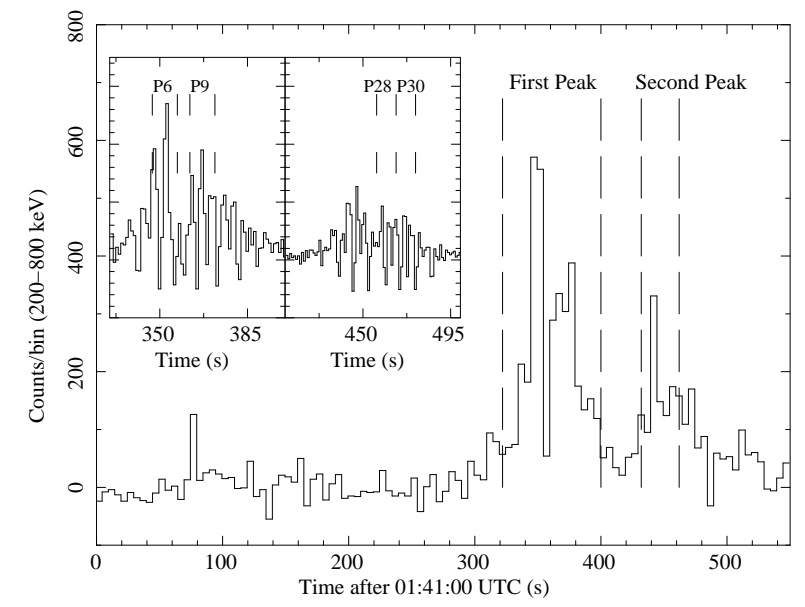

Fig. 1.- Compton light curve of GRB 041219A. Bin size is $5 \mathrm{~s}$. The two insets show a magnified view of the two peaks, binned at $1 \mathrm{~s}$. The analyzed intervals, are shown with dashed lines. P8 is omitted for clarity.

share was saturated, and hence not all the events could be sent to the ground. Images based on Compton events selected in the first and second peak show the bursting source respectively at a 32 sigma level and 20 sigma level. An image made on the whole GRB is shown in Fig 2, and the position derived $\left(\alpha_{J 2000}=00^{h} 24^{m} 24^{s}, \delta_{J 2000}=\right.$ $+62^{\circ} 49^{\prime} 44^{\prime \prime}$ with an uncertainty of $1^{\prime}$ at $90 \%$ c.l.) is consistent with the one derived from simultaneous optical data (Vestrand et al. 2005).

To measure the polarization, we followed the same procedure described in Forot et al. (2008) that allowed to successfully detect a polarized signal from the Crab nebula. To derive the source flux as a function of $\phi$, the Compton photons were divided in 6 bins of $30^{\circ}$ as a function of the azimuthal scattering angle. To improve the signalto-noise ratio in each bin, we took advantage of the $\pi$-symmetry of the differential cross section (see Eq. 1), i.e. the first bin contains the photons with $0^{\circ}<\phi<30^{\circ}$ and $180^{\circ}<\phi<210^{\circ}$, etc. Then the chance coincidences (i.e. photons interacting in both detectors, but not related to a Compton event), have been subtracted from each detector image following the procedure described in Forot et al. (2008). The derived detector images were then deconvolved to obtain sky images, where the flux of the source in each bin is measured by fitting the instrumental PSF to the source

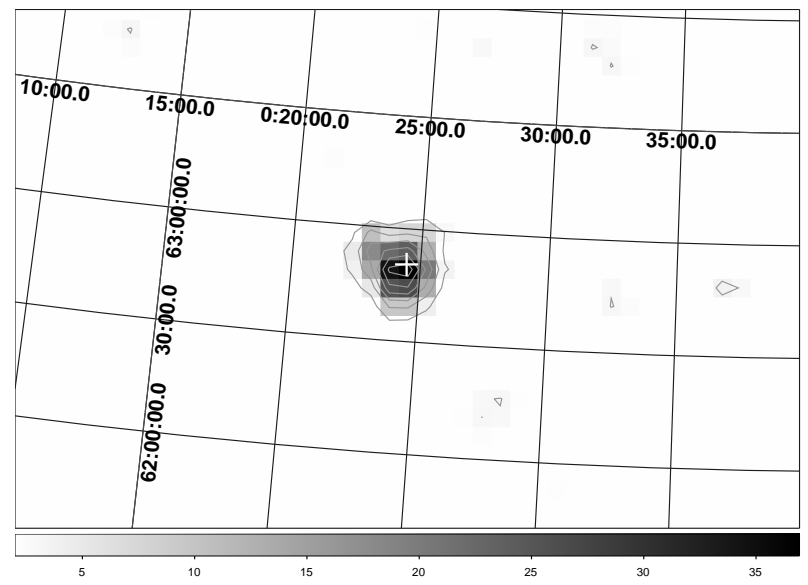

Fig. 2.- 200-800 keV Compton significance image of GRB 041219A. The source is detected at 37 sigma level. The white cross indicates the position of the optical counterpart (Vestrand et al. 2005), and grey contours are traced at equal significance starting from 3 in steps of 6 .

peak. We finally fitted using a least squares technique the polarigrams (see Fig. 3) with Eq. 2 to derive $a_{0}$ and $\phi_{0}$, and the errors on the parameters are dominated by the statistics of the data points. To evaluate the goodness of our fits, we computed the chance probability (see Eq. 2 in Forot et al. 2008) that our polarigrams are due to an unpolarized signal, and reported these values in Fig. 3.

\section{Results}

We analyzed the different portions of the GRB, focusing on the brightest parts. First we analyzed the entire first and second peak, and then we performed a time resolved analysis: 36 intervals lasting $10 \mathrm{~s}$, each one overlapping for $5 \mathrm{~s}$ with the previous one, have been analyzed over the whole duration of the GRB starting at 01:46:22 U.T. until 01:49:22 U.T. The most significant nonoverlapping ones (P6, P8, P28, P30) have been chosen for the polarization analysis 1 . The integration times and the imaging significance of the chosen time intervals are reported in Table1, and

\footnotetext{
${ }^{1}$ Even if P9 is more significant than P8 (see Table 1), we prefer to discuss P8 because it is simultaneous to the SPI analysis. Anyway, P8 and P9 results are statistically consistent.
} 
Fig. 1. The azimuthal distributions of the GRB flux for the different time intervals are reported in Fig. 3.

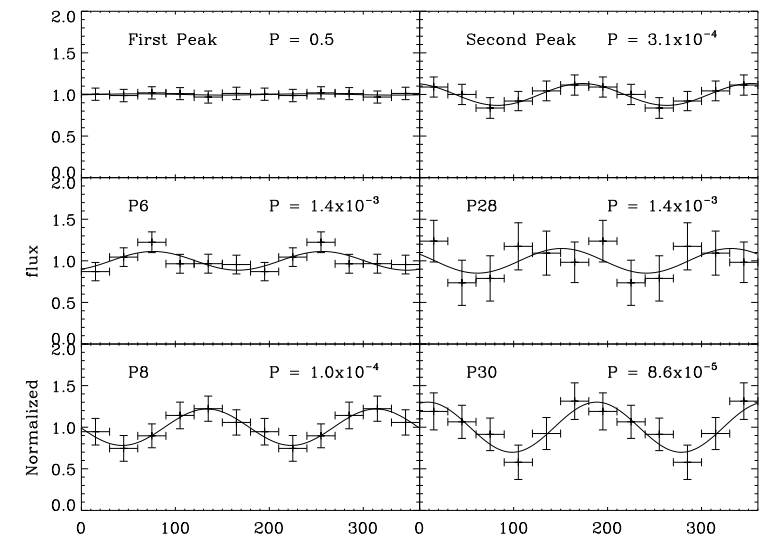

Fig. 3.- Polarigrams of the different time intervals that have been analysed (see Table 1). For comparison purposes, the curves have been normalized to their average flux level. The crosses represent the data points (replicated once for clarity) and the continuous line the fit done using Eq. 2. The chance probability of a non-polarized signal is reported in each panel.

As can be seen, no polarization signal could be found integrating over the whole first peak, and the upper limit is $4 \%$. On the other hand, a modulated signal is seen in the second peak corresponding to $\Pi=43 \pm 25 \%$. Integrating over smaller portions of the GRB, we measure highly polarized signals, especially in P8, P9 and P30.

\section{Discussion and conclusions}

Using SPI data McGlynn et al. (2007) reported high polarization for GRB 041219A, but they restricted their analysis to the first peak of the GRB. They analyzed a $66 \mathrm{~s}$ interval within the first peak, and a $12 \mathrm{~s}$ interval on the brightest part of it starting at 01:46:54 U.T. The latter corresponds to our P8 interval and indeed our results are compatible at 1 sigma level, both for the polarization fraction and angle, for which they find $\Pi=68 \pm 29 \%$ and $P A=70_{-10^{\circ}}^{+14^{\circ}}(100 \mathrm{keV}-1 \mathrm{MeV})$. On the other hand we cannot confirm their result on the broader 66 s time interval $\left(\Pi=26 \pm 20 \%\right.$ and $P A=70_{-27^{\circ}}^{+19^{\circ}}$, starting at 01:46:21 U.T., which corresponds to
Table 1: Polarization results for the different time intervals.

\begin{tabular}{cccccc}
\hline \hline Name & $\begin{array}{c}\mathrm{T}_{\text {start }} \\
\text { U.T. }\end{array}$ & $\begin{array}{c}\mathrm{T}_{\text {stop }} \\
\text { U.T. }\end{array}$ & $\begin{array}{c}\Pi \\
\%\end{array}$ & $\begin{array}{c}P A \\
\text { degrees }\end{array}$ & $\begin{array}{c}\text { Image } \\
\text { SNR }\end{array}$ \\
\hline First Peak & $01: 46: 22$ & $01: 47: 40$ & $<4$ & - & 32.0 \\
Second Peak & $01: 48: 12$ & $01: 48: 52$ & $43 \pm 25$ & $38 \pm 16$ & 20.0 \\
P6 & $01: 46: 47$ & $01: 46: 57$ & $22 \pm 13$ & $121 \pm 17$ & 21.5 \\
P8 & $01: 46: 57$ & $01: 27: 07$ & $65 \pm 26$ & $88 \pm 12$ & 15.9 \\
P9 & $01: 47: 02$ & $01: 47: 12$ & $61 \pm 25$ & $105 \pm 18$ & 18.2 \\
P28 & $01: 48: 37$ & $01: 48: 47$ & $42 \pm 42$ & $106 \pm 37$ & 9.9 \\
P30 & $01: 48: 47$ & $01: 48: 57$ & $90 \pm 36$ & $54 \pm 11$ & 11.8 \\
\hline
\end{tabular}

Errors are given at $1 \sigma$ c.l. for one parameter of interest.

our first peak analysis, where we do not detect any polarized signal. By comparing $\mathrm{P} 6$ and $\mathrm{P} 8$, it seems that the polarization signal in this time interval results from the superposition of signals with different polarization angles, which could give rise to a null signal over a longer time interval. The shorter duration of the second pulse, where a polarization signal (P30) is clearly dominant over the others, could, on the other hand, explain the detection of a polarized signal over the whole second peak. Similarly, the $12 \mathrm{~s}$ peak emission may dominate the SPI data, while in the IBIS data this time period is heavily affected by telemetry losses, making the flux ratio between the brightest part (P8) and the rest of the GRB first peak smaller. In other words, the different polarization phases of the rest of the peak have a larger weight in our average measure.

The expected level of polarization of the prompt $\gamma$-ray emission in GRBs has been estimated by several authors for different models, or variations within them. In most cases, the observed $\gamma$-ray emission is due to the synchrotron radiation from relativistic electrons in the fast cooling regime. Their time-averaged distribution is a broken power law, $n(\gamma) \propto \gamma^{-p^{\prime}}$ with $p^{\prime}=p+1$ above $\Gamma_{\mathrm{m}}$ and $p^{\prime}=2$ below, where $\Gamma_{\mathrm{m}}$ is the minimum Lorentz factor of the injected distribution of electrons, and $p \simeq 2-2.5$ its slope (Sari, Piran \& Narayan 1998). The intrinsic polarization of the synchrotron radiation, $\Pi_{\mathrm{svn}}=\left(p^{\prime}+1\right) /\left(p^{\prime}+7 / 3\right)$ (Ribicky \& Lightman 1979) is then of the order of 
$\Pi_{\text {syn }}=(p+2) /(p+10 / 3) \simeq 75 \%$ above $\nu_{\mathrm{m}}$ and $\Pi_{\text {syn }}=9 / 12 \simeq 70 \%$ below, where $\nu_{\mathrm{m}}$, the peak of the spectrum in $\nu F_{\nu}$, is the synchrotron frequency of electrons at $\Gamma_{\mathrm{m}}$. High polarization levels can also be reached if inverse Compton scatterings are the dominant radiative process.

Our results show that (i) the polarization level in GRB04119A is varying on short time scales and can reach high values that correspond to a sizeable fraction of the intrinsic polarization $\Pi_{\text {syn }}$. The polarization angle is varying as well, and (ii) the time-averaged value over longer intervals shows reduced polarization. We discuss these results in the context of different scenarios :

(1) synchrotron emission from shock-accelerated electrons in a relativistic jet with an ordered magnetic field contained in the plane perpendicular to the jet velocity. This geometry is favored if the field is carried by the outflow from the central source, as the poloidal component decreases much faster with radius than the toroidal one. The polarization level at the peak of a given pulse can be as high as $\Pi / \Pi_{\text {syn }} \sim 0.8$, i.e. $\Pi \sim 60 \%$, leading to a maximum time-averaged polarization in long intervals of $\Pi / \Pi_{\max } \sim 0.6$, i.e. $\Pi \sim 45 \%$ in this case (Granot 2003; Granot \& Königl 2003; Nakar. Piran \& Waxman 2003). The main requirement is to have a uniform magnetic field in space, i.e. with a coherent scale $R \theta_{\mathrm{B}}$ with $\theta_{\mathrm{B}} \gtrsim 1 / \Gamma$. The fact that the polarization level and angle vary during the burst indicates on the other hand that the field is not necessarily uniform in time, as it would be required to explain a high level of the time-integrated polarization (Nakar. Piran \& Waxman 2003). A magnetic field anchored in the central engine and carried by the outflow to large distance (see e.g. Spruit et al. 2001) is therefore not the only possibility. A magnetic field generated at the shock could also work, and even favor variability, if there is a process capable to increase the field coherence scale (the field is most probably initially generated on small, skin-depth, scales). The existence of such a process is unclear in our present knowledge of the micro-physics in mildly relativistic shocks (see e.g. Keshet et al. 2008). Note that the condition $\theta_{\mathrm{B}} \gtrsim 1 / \Gamma$ is really necessary only in the pulses with the highest level of polarization. If $\theta_{\mathrm{B}}$ is smaller, so that a number $N \sim\left(\Gamma \theta_{\mathrm{B}}\right)^{-2}$ of mutu- ally incoherent patches are present is the visible region, the level of polarization will decrease, but the variability (both of the polarization level and angle) will increase (Granot 2003). If the radiating electrons are accelerated in internal shocks (Rees \& Mészáros 1994; Kobavashi. Piran \& Sari 1997; Daigne \& Mochkovitch 1998), the Lorentz factor is necessarily varying in the outflow, which can be an additional source of variability for the polarization. If $\theta_{\mathrm{B}}$ and $1 / \Gamma$ are close, the number of coherent patches in the visible region could vary from a pulse to another. We therefore conclude that any scenario where the observed gamma-rays are produced by synchrotron radiation from electrons in a relativistic jet with an ordered magnetic field in the plane perpendicular to the jet velocity seems fully consistent with our observations, as long as the coherence scale $\theta_{\mathrm{B}}$ of the field is larger than $1 / \Gamma$ in most of the emitting regions. A potential difficulty remains: an additional random component of the magnetic field is probably necessary to allow for particle acceleration in shocks. This component would reduce the level of polarization by a factor that is however difficult to estimate, as the intensity of the the former is not well constrained Granot 2003; Nakar, Piran \& Waxman 2003);

(2) synchrotron emission from a purely electromagnetic outflow. The estimated level of polarization is comparable with the previous scenario (Lyutikov et al. 2003). In addition, a magnetic field with a large coherence scale is naturally expected in such a purely electromagnetic outflow. One potential difficulty is, however, related to the mechanism responsible for the energy dissipation. It this scenario, the energy has to be extracted from the magnetic field before being radiated. Therefore magnetic dissipation must occur in the emitting region, changing the field geometry, which becomes probably much less ordered, reducing the final level of polarization by a large factor (Lvutikov et al. 2003; Nakar. Piran \& Waxman 2003). This effect is however difficult to estimate, as the details of the physical processes that could lead to magnetic dissipation in such an outflow are still far from being understood;

(3) synchrotron emission from shock-accelerated electrons in a relativistic jet with a random field 
generated at the shock and contained in the plane perpendicular to the jet velocity. A high level of polarization can be obtained even with a random magnetic field if the jet is observed from just outside its edge (Ghisellini \& Lazzati 1999; Waxman 2003). The polarization at the peak of a given pulse can reach $\Pi / \Pi_{\text {syn }} \simeq 0.8$, i.e. $\Pi \simeq 60 \%$ resulting in a time-integrated value of the order of $\Pi / \Pi_{\mathrm{syn}} \simeq 0.5-0.6$, i.e. $\Pi \simeq 40-45 \%$ (Granot 2003; Granot \& König] 2003; Nakar, Piran \& Waxman 2003). However these high values are obtained if the jet is seen with $\theta_{\text {obs }} \simeq \theta_{\mathrm{j}}+1 / \Gamma$, where $\theta_{\mathrm{j}}$ is the opening angle of the jet and $\theta_{\text {obs }}$ the angle between the line-ofsight and the jet axis. Such viewing conditions are rare, except if $\theta_{\mathrm{j}} \sim 1 / \Gamma$. Variability of the polarization level is expected if the Lorentz factor is varying in the outflow, as for instance in the internal shock model. Different pulses in the light curve correspond to viewing angles $\theta_{\mathrm{obs}}=\theta_{\mathrm{j}}+k / \Gamma$, where $k$ is larger for emitting regions with a larger Lorentz factor. The highest polarization is obtained in pulses with $k \sim 1$ whereas the pulse flux decreases with $k$ for $k \geq 0$. The highest polarization should therefore not be found in the brightest pulses, which is difficult to test as all pulses do not have necessarily the same intrinsic luminosity. In addition, when several pulses superimpose, the measured polarization level, which is flux-weighted, could be reduced by a sizeable factor (Granot \& Königl 2003). Finally, the observed polarization can also be reduced if the jet edges are not sharp enough (Nakar, Piran \& Waxman 2003). We conclude that this model cannot be rejected as it can in principle reach high levels of polarization, but the conditions necessary for it seem more difficult to be achieved than in model (1). As shown by Granot \& Königl (2003), similar conditions as for scenario (3) would be required for a scenario where the field is ordered but parallel to the jet, leading to the same conclusions.

(4) inverse Compton emission from relativistic electrons in a jet propagating within a photon field ("Compton drag" model). The level of polarization in this scenario can be even higher than for the synchrotron radiation and reach $60-100 \%$, but only under the condition that the jet is narrow with $\Gamma \theta_{\mathrm{j}} \lesssim 5$ (Lazzati et al. 2004). The maximum level of polarization is again obtained for $\theta_{\text {obs }} \simeq \theta_{\mathrm{j}}+1 / \Gamma$. These viewing conditions are very similar to those of models (3) and are discussed below in the case of GRB 041219A. Again, the polarization is reduced if the edges of the jet are not sharp enough. Variability of the Lorentz factor will again result in a varying polarization, with the same difficulties regarding the final level of polarization than in model (3). However, variations of the Lorentz factor could possibly be less large in this scenario as part of the variability of the light curve can be related to the inhomogeneity of the ambient photon field.

Combining the Yonetoku relation (Yonetoku et al. 2004) to estimate the redshift and the isotropic energy, with the standard energy reservoir derived by Frail et al. (2000), McGlynn et al. (2007) have estimated that $\theta_{\mathrm{j}} \sim 2-3$ degrees in GRB 041219A, which leads to $\Gamma \theta_{\mathrm{j}} \sim(3.5-5)(\Gamma / 100)$. Such an estimate, based on very debated relations, indicates that the peculiar viewing conditions necessary in the geometric models are difficult in the case of GRB 041219A but cannot be fully excluded. Models (3-4) can therefore not be rejected on the basis of these conditions only. As explained above, it is however not clear if a high polarization level can be maintained together with a high time variability. The status of model (2) is not clear as the details of the magnetic dissipation process are crucial and not well understood. Finally, our results favor model (1) as it seems to have the best capabilities to maintain a high polarization level with some variability. An additional random component of the magnetic field is however necessary for particle acceleration at the shock and its negative impact on the level of polarization should be carefully estimated. A final answer to distinguish between intrinsic and geometric models could be obtained by accumulating more observations. Indeed, models (1-2) predict a polarized emission for all bursts, whereas models (3-4) would predict that only a small fraction of GRBs are highly polarized. This idea has been tested recently by Montecarlo simulations performed by Toma et al. (2008), who conclude that if more than $30 \%$ of bursts are polarized, geometric models can be ruled out. This shows the importance of polarimetric measurements for the understanding of intrinsic properties of GRBs, but the current instrumentation is statistically limited 
and can provide measurements just for the brightest events. To significantly increase the sample of GRBs with measured polarization, future GRB mission with wide field of views and polarimetric capabilities are needed.

D.G. acknowledges the French Space Agency (CNES) for financial support. ISGRI has been realized and maintained in flight by CEA-Saclay/Irfu with the support of CNES. Based on observations with INTEGRAL, an ESA project with instruments and science data centre funded by ESA member states with the participation of Russia and the USA.

\section{REFERENCES}

Coburn, W., \& Boggs, S.E. 2003, Nature, 423, 415

Daigne, F., \& Mochkovitch, R. 1998, MNRAS, 296,275

Forot, M., et al. 2008, ApJ, 688, L29

Frail D. et al., 2001, ApJ, 562, L55

Ghisellini, G. and Lazzati, D., 1999, MNRAS, 309, L7

Gruzinov, A. 1999, ApJ, 525, L29

Gruzinov, A., \& Waxman E., 1999, ApJ, 511, 852

Granot, J., 2003, ApJ, 596, L17

Granot, J. and Königl, A., 2003, ApJ, 594, L83

Keshet, U., Katz, B., Spitkovsky, A. and Waxman, E., 2008, arXiv:astro-ph/0802.3217

Kobayashi, S. and Piran, T. and Sari, R., 1997, ApJ, 490, 92

Labanti, C., Di Cocco, G., Ferro, G., et al. 2003, A\&A, 411, L149

Lazzati, D., et al. 2004, MNRAS, 347, L1

Lazzati, D. 2006, New Journal of Physics, 8, 131

Lebrun, F., Leray, J.P., Lavocat, P., et al. 2003, A\&A, 411, L141

Lei, F., et al. 1997, Space Science Reviews, 82, 309

Lyutikov, M. Pariev, V.I., \& Blandford, R.D. 2003, ApJ, 597, 998
Lyutikov, M. 2006, New Journal of Physics, 8, 199

McBreen, S., Hanlon, L., McGlynn, S., et al. 2006, A\&A, 455, 433

McGlynn, S., Clark, D.J., Dean, A.J., et al. 2007, A\&A, 466, 895

Mészáros, P. , 2006, Reports on Progress in Physics, 69, 2259

Nakar, E; and Piran, T. and Waxman, E., 2003, JCAP, 10, 005

Rees, M. J. and Mészáros, P. 1994, ApJ, 430, L93

Ribicky, G.B., \& Lightman, A.P. 1979, Radiative Processes in Astrophysics (New York: WileyInterscience)

Rutledge, R.E., \& Fox, D.E. 2004, MNRAS, 350, 1288

Sari, E. and Piran, T. and Narayan, R., 1998, ApJ, 497, L17

Spruit, H.C., Daigne, F., \& Drenkhahn, G. 2001, A\&A, 369, 694

Toma, K. et al., 2008, submitted to ApJ, arXiv:astro-ph/0812.2483

Ubertini, P., Lebrun, F., Di Cocco, G., et al. 2003, A\&A, 411, L131

Vedrenne, G., Roques, J.-P., Schönfelder, V., et al. 2003, A\&A, 411, L63

Vestrand, W. T., Wozniak, P. R., Wren, J. A., et al. 2005, Nature, 435, 768

Vianello, G., Götz, D., \& Mereghetti, S. 2008, A\&A, in press, arXiv:astro-ph/0812.3349

Yonetoku, D. et al., 2004, ApJ, 609, 935

Waxman, E. 2003, Nature, 423, 388

Wigger, C., et al. 2004, ApJ, 613, 1088

Willis, D.R., Barlow, E.J., Bird, A.J., et al. 2005, A\&A, 439, 245

Winkler, C., Courvoisier, T.J.-L., Di Cocco G., et al. 2003 A\&A, 411, L1

This 2-column preprint was prepared with the AAS LATEX macros v5.2. 